\title{
Immunobullous disease
}

\author{
Authors: Thomas J Tull ${ }^{\mathrm{A}}$ and Emma Benton ${ }^{\mathrm{B}}$
}

Immunobullous diseases are blistering cutaneous disorders that are caused by pathogenic antibodies binding to protein targets within the skin. There are a range of immunobullous disorders with characteristic morphology that relates to the structural properties of the target protein. In this article we will describe the pathogenesis, clinical features and treatment of the most common immunobullous disorders.

\section{Pemphigus}

Pemphigus is a group of diseases caused by antibodies that bind desmoglein (Dsg) 1 and 3, which are cadherin proteins found within desmosomes. Pathogenic anti-Dsg antibodies cause a loss of keratinocyte adhesion resulting in acantholysis (separation of keratinocytes) and intraepithelial blister formation. ${ }^{2}$ The incidence of pemphigus varies between $0.5-34$ cases/million/year and there is significant geographic variation in incidence rates. ${ }^{3}$ Pemphigus vulgaris (PV) is the most common variant in most countries and is characterised by anti-Dsg-3 and/or Dsg-1 antibodies. Dsg-1 is expressed predominantly in the uppermost layers of the epidermis while Dsg-3 is expressed predominantly in mucosal tissues but also in the lower epidermis. PV can therefore be predominantly mucosal in which anti-Dsg-3 antibodies are present, or mucocutaneous where both anti-Dsg-1 and 3 antibodies are present. Intraepithelial acantholysis causes flaccid blisters and shallow erosions that are most common on the head, upper trunk, axillae and groin (Figs 1a and b). Mucosal lesions are characterised by irregularly shaped erosions that are most commonly seen on the buccal mucosa, palate and the gingiva (Figs 1c and d). ${ }^{2}$ Laryngeal, oesophageal, conjunctival and genital lesions can also occur. Pemphigus foliaceus (PF) is characterised by anti-Dsg-1 antibodies and therefore only affects the skin and not mucosal sites. Crusted erosions are seen predominantly in seborrheic areas such as the central chest and back, face and scalp (Figs $2 a$ and b). Endemic PF, otherwise known as fogo selvagem, is common in South America and results in a similar clinical picture, although is thought to be acquired following insect bites due to cross reactivity of Dsg-1 and salivary gland antigens of the sand fly. ${ }^{4}$ Paraneoplastic pemphigus (PNP) is a rare variant that results in severe mucocutaneous disease and is most commonly associated with non-Hodgkin lymphoma, Castleman's disease and chronic lymphocytic leukaemia. ${ }^{5}$

Authors: Aspecialist registrar in dermatology, St John's Institute of Dermatology, London, UK; ${ }^{\text {}}$ consultant dermatologist, St John's Institute of Dermatology, London, UK
A biopsy of perilesional skin is the most reliable method for the diagnosis of pemphigus. Haematoxylin and eosin (H\&E) staining demonstrates acantholysis of keratinocytes in the lower epidermis and direct immunofluorescence (IMF) identifies immunoglobulin (Ig) G deposition within the epidermis (Fig 2c). Serum anti-Dsg antibodies can be identified by indirect IMF or ELISA, the latter being a useful tool for monitoring disease activity as anti-Dsg titres lower as disease improves.

Initial treatment involves the induction of disease remission with corticosteroids. Oral prednisolone at a dose of $0.5 \mathrm{mg} / \mathrm{kg}$ or $1 \mathrm{mg} / \mathrm{kg}$ for severe cases is recommended. ${ }^{6}$ Disease remission is then maintained with azathioprine, mycophenolate mofetil (MMF) or cyclophosphamide. Refractory cases can be treated with intravenous immunoglobulin or the anti-CD20 biologic agent rituximab, although evidence is emerging that rituximab may be an effective agent for the induction of disease remission. ${ }^{7}$ Veltuzumab is a recently developed anti-CD20 biologic agent which can be administered subcutaneously and has been used for

\section{Key points}

Immunobullous skin diseases arise due to pathogenic antibodies that bind targets in the epidermis, epidermal basement membrane or dermis.

Antibody binding within the epidermis results in flaccid blisters and erosions while binding to the epidermal basement membrane causes tense bullae.

Biopsy of perilesional skin for direct immunofluorescence is the gold standard investigation for the diagnosis of immunobullous skin diseases.

Bullous pemphigoid (BP) has been associated with dipeptidyl-peptidase 4 inhibitors such as linagliptin. These agents should be discontinued if a diagnosis of BP is confirmed.

A gluten free diet is essential for the treatment of dermatitis herpetiformis and reduces the risk of developing gastrointestinal lymphoma.

KEYWORDS: immunobullous disorders, pemphigus, bullous pemphigoid, epidermolysis bullosa aquisita, dermatitis herpetiformis

DOI: 10.7861/clinmed.2021-0232 

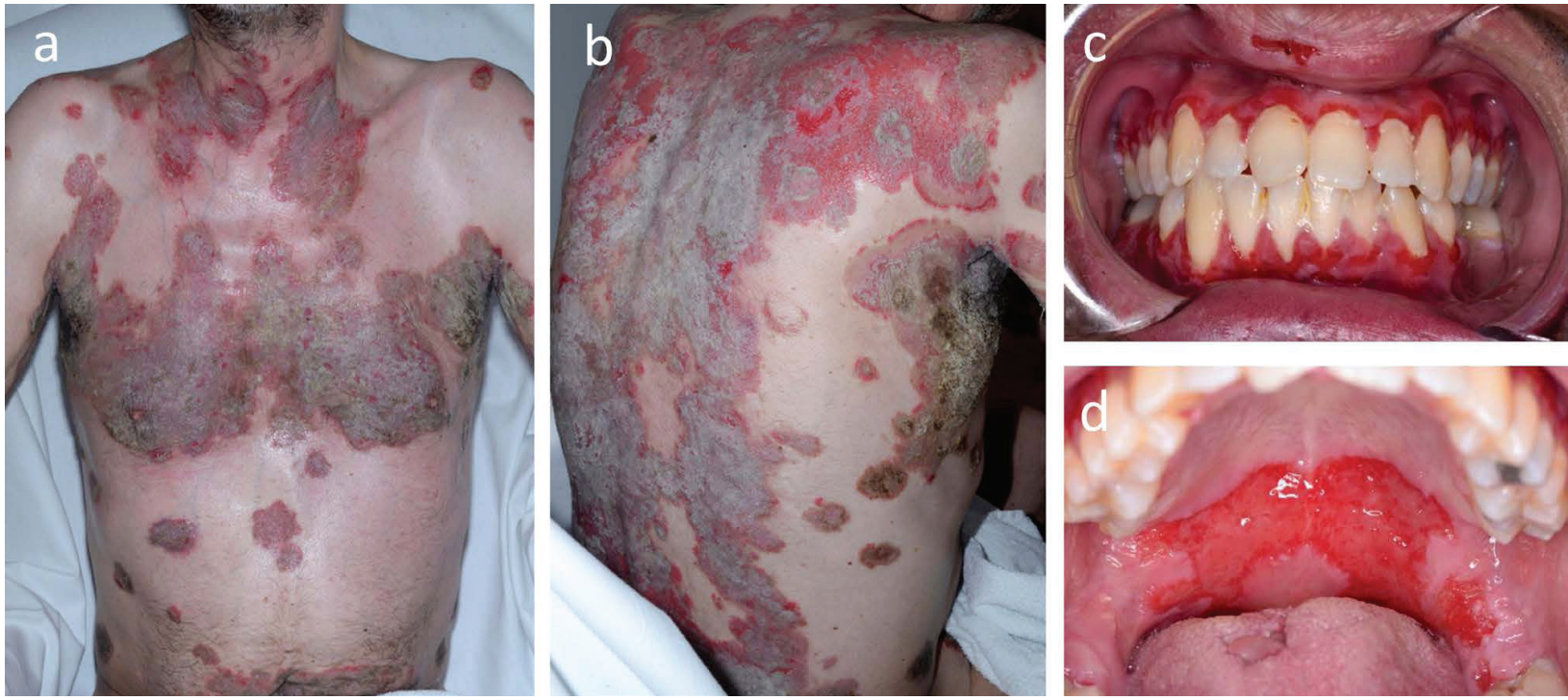

Fig 1. Pemphigus vulgaris. $a$ and b) Superficial erosions on the chest and back. c) Erosive gingivitis. d) Irregular shallow erosions on the soft palate.

refractory pemphigus. ${ }^{8}$ Treatments under development include the use of CAR-T cells to selectively remove Dsg reactive B cells, competitive inhibition of $B$ cell activating factor receptor using ianalumab and suppression of B cell activation using Bruton kinase inhibitors. 2,9

\section{Bullous pemphigoid}

Bullous pemphigoid (BP) results from antibodies binding to bullous pemphigoid antigens 180 (BP180) and 230 (BP230) which are hemidesmosomal proteins found within the basement membrane of the epidermis. Antibody binding results in subepithelial blister formation. BP tends to affect patients over the age of 70 and has an incidence of $2.4-23$ cases/million/year. ${ }^{10}$ The pathogenesis for the majority of cases is likely to represent a genetic predisposition and environmental factors. ${ }^{2} \mathrm{BP}$ is, however, associated with neurodegenerative disease such as multiple sclerosis and Alzheimer's dementia. ${ }^{11}$ As BP antigens are expressed in central nervous system tissue this has led to the hypothesis of cross-reactivity between central nervous system and cutaneous antigens.

An association of BP with dipeptidyl-peptidase 4 (DPP-4) inhibitors such as linagliptin that are used to treat diabetes has also recently emerged through case reports and pharmacovigilance database analyses. ${ }^{12}$ The association is stronger in males and patients over the age of 70 and case control studies have estimated a three-fold risk of developing BP on DPP-4 inhibitors..$^{12}$ The pathophysiology underlying this association is not understood but it has been postulated that DPP-4 inhibitors may reduce plasmin mediated BP180 cleavage thereby affecting its antigenicity. ${ }^{13}$ If BP is suspected then DPP- 4 inhibitors should be discontinued as this can result in disease regression.
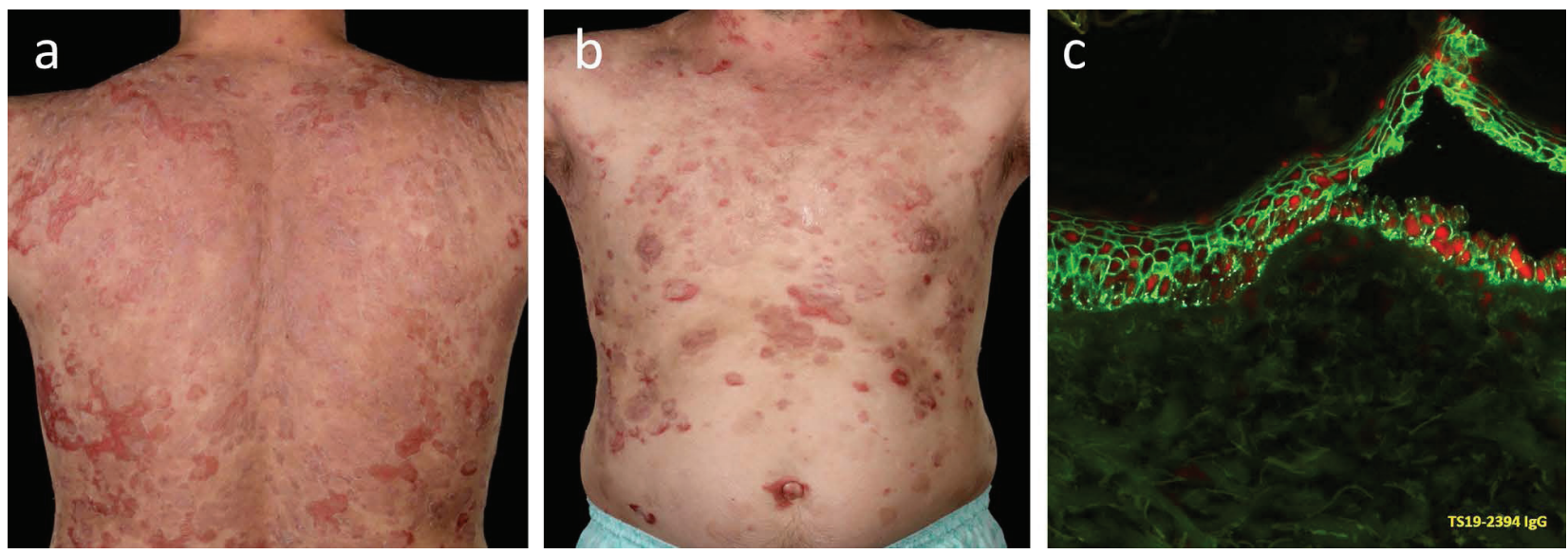

Fig 2. Pemphigus foliaceus. $a$ and b) Shallow scaly erosions on the back and chest. c) Direct immunofluorescence demonstrating intraepithelial immunoglobulin $\mathrm{G}$ binding as evidenced by fluorescein isothiocyanate (green) fluorescent signal with an intraepithelial blister formation to the right of the image. 


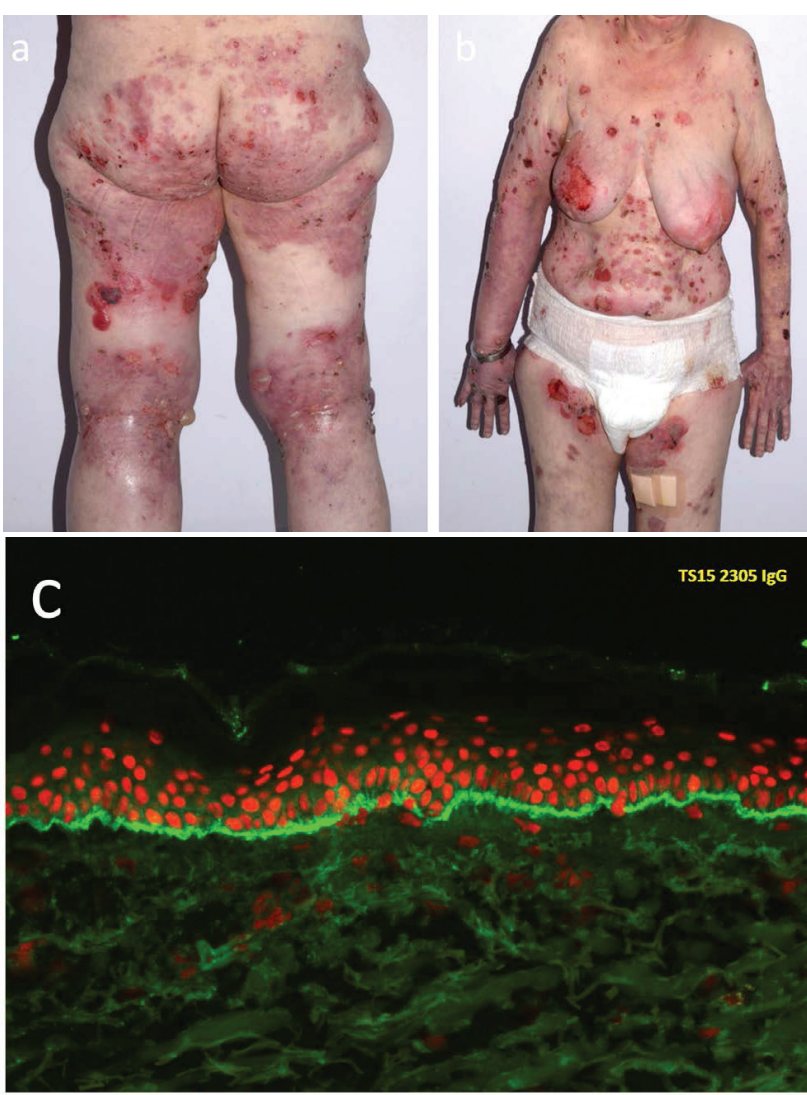

Fig 3. Bullous pemphigoid (BP). a) and b) Tense bullae, erosions and urticated lesions on the trunk and limbs. c) Direct immunofluorescence demonstrating immunoglobulin $\mathrm{G}$ binding to the basement membrane of the epidermis which is characteristic of BP.

Clinically, BP is characterised by subepidermal blistering resulting in tense bullae that are preceded by itchy erythematous and urticated plaques (Figs $3 a$ and b). Mucosal ulceration is less common and occurs in approximately $15 \%$ of patients. ${ }^{14}$ Diagnosis is by perilesional skin biopsy. H\&E staining typically demonstrates an inflammatory cell infiltrate in the upper dermis rich in eosinophils and direct IMF demonstrates IgG and C3 deposition at the epidermal basement membrane (Fig 3c). Anti-BP180 and antiBP230 antibodies can also be detected by ELISA. First-line treatment is usually with an oral corticosteroid, such as prednisolone at a dose of $0.3-1 \mathrm{mg} / \mathrm{kg}$ depending on disease severity with azathioprine, MMF, methotrexate or cyclophosphamide. ${ }^{15}$ Doxycycline has antiinflammatory effects and can be effective in patients in whom prednisolone and immunosuppression is not appropriate. ${ }^{16}$ As with pemphigus, intravenous IG and rituximab can be used for treatment refractory cases. BP is usually a self-limiting disease with an average disease duration of 3-6 years.

\section{Epidermolysis bullosa aquisita}

Epidermolysis bullosa aquisita (EBA) is a rare subepidermal blistering condition that arises due to antibodies binding to type VII collagen, which is found in the anchoring fibrils that link the epidermal basement membrane to dermal extracellular matrix proteins. It can be divided into classical or inflammatory EBA.

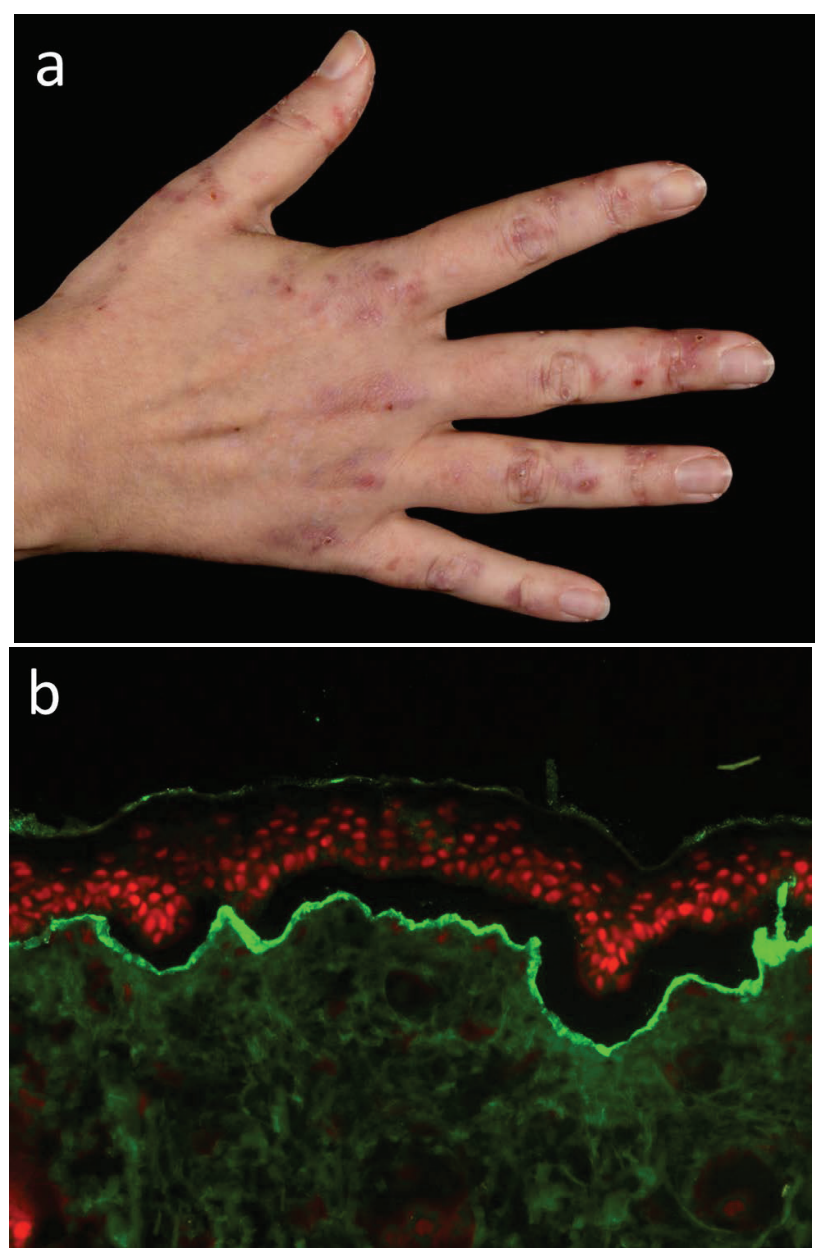

Fig 4. Epidermolysis bullosa aquisita. a) Superficial erosions and scarring on the right dorsal hand. b) Direct immunofluorescence of salt split skin showing immunoglobulin $\mathrm{G}$ binding to the base of the subepidermal blister.

Classical EBA is characterised by skin fragility and tense bullae or erosions at the sites of mechanotrauma, such as the hands, elbows and knees (Fig 4a). Inflammatory EBA is characterised by tense bullae and mucosal ulceration and mimics BP and mucous membrane pemphigoid. Mucosal involvement can be extensive and involve the pharynx, larynx and oesophagus resulting in dysphagia, dysphonia and oesophageal strictures. Such patients require a multidisciplinary approach to their management.

EBA is diagnosed by direct IMF using a salt split skin technique which separates the epidermal basement membrane. This identifies IgG binding on the floor of the split and helps distinguish it from BP where binding is to the roof (Fig 4b). ELISA can also be used to identify type VII collagen antibodies. Treatment is similar to BP, although EBA may be more difficult to supress.

\section{Dermatitis herpetiformis}

Dermatitis herpetiformis $(\mathrm{DH})$ is a cutaneous manifestation of coeliac disease (CD) and arises due to pathogenic IgA transglutaminase (TG) antibody binding within the skin. Both $\mathrm{DH}$ and $C D$ share the same human leukocyte antigen haplotypes DQ2 and DQ8, and the vast majority of patients with DH have 
endoscopic evidence of $C D .{ }^{17} \mathrm{DH}$ is characterised by itchy vesicles or crusted erosions with a predilection for the extensor forearms, elbows and buttocks. Granular IgA deposition in the upper dermis is seen on direct IMF and is diagnostic. Anti-TG antibodies can also be demonstrated by ELISA and have replaced anti-gliadin antibodies in the diagnosis of $\mathrm{DH} .^{18}$ The first-line treatment is a gluten free diet which is essential to reduce the long-term risk of developing gastrointestinal lymphoma. ${ }^{19} \mathrm{DH}$ may take many months to resolve and additional treatment with dapsone may be required. Rarely $\mathrm{DH}$ can be refractory to a gluten free diet, although in reported cases, there was no endoscopic evidence of $\mathrm{CD}$ and none developed lymphoma. ${ }^{20}$

\section{Acknowledgements}

We are grateful to Dr John Mee for providing immunofluorescence images for this article.

\section{References}

1 Amagai M, Klaus-Kovtun V, Stanley JR. Autoantibodies against a novel epithelial cadherin in pemphigus vulgaris, a disease of cell adhesion. Cell 1991;67:869-77.

2 Egami S, Yamagami J, Amagai M. Autoimmune bullous skin diseases, pemphigus and pemphigoid. J Allergy Clin Immunol 2020;145:1031-47.

3 Jelti, L, Cordel N, Gillibert A et al. Incidence and mortality of pemphigus in France. J Invest Dermatol 2019;139:469-73.

4 Qian Y, Jeong JS, Abdeladhim M et al. IgE anti-LJM11 sand fly salivary antigen may herald the onset of fogo selvagem in endemic Brazilian regions. J Invest Dermatol 2015;135:913-5.

5 Kaplan I, Hodak E, Ackerman L et al. Neoplasms associated with paraneoplastic pemphigus: a review with emphasis on nonhematologic malignancy and oral mucosal manifestations. Oral Oncol 2004;40:553-62.

6 Harman KE, Brown D, Exton LS et al. British Association of Dermatologists' guidelines for the management of pemphigus vulgaris 2017. Br J Dermatol 2017;177:1170-201.

7 Joly P, Maho-Vaillant M, Prost-Squarcioni C et al. First-line rituximab combined with short-term prednisone versus prednisone alone for the treatment of pemphigus (Ritux 3): a prospective, multicentre, parallelgroup, open-label randomised trial. Lancet 2017;389:2031-40.

8 Ellebrecht, CT, Choi EJ, Allman DM et al. Subcutaneous veltuzumab, a humanized anti-CD20 antibody, in the treatment of refractory pemphigus vulgaris. JAMA Dermatol 2014;150:1331-5.
9 Ellebrecht, CT, Bhoj VG, Nace A et al. Reengineering chimeric antigen receptor T cells for targeted therapy of autoimmune disease. Science 2016;353:179-84.

10 Kridin K, Ludwig RJ. The growing incidence of bullous pemphigoid: overview and potential explanations. Front Med (Lausanne) 2018;5:220.

11 Forsti AK, Huilaja L, Schmidt E, Tasanen K. Neurological and psychiatric associations in bullous pemphigoid-more than skin deep? Exp Dermatol 2017;26:1228-34.

12 Kridin K, Bergman R. Association of bullous pemphigoid with dipeptidyl-peptidase 4 inhibitors in patients with diabetes: estimating the risk of the new agents and characterizing the patients. JAMA Dermatol 2018;154:1152-8.

13 Izumi K, Nishie W, Mai Y et al. Autoantibody Profile Differentiates between inflammatory and noninflammatory bullous pemphigoid. J Invest Dermatol 2016;136:2201-10.

14 della Torre R, Combescure C, Cortes B et al. Clinical presentation and diagnostic delay in bullous pemphigoid: a prospective nationwide cohort. Br J Dermatol 2012:167:1111-7.

15 Venning VA, Taghipour K, Mohd Mustapa MF, Highet AS, Kirtschig G. British Association of Dermatologists' guidelines for the management of bullous pemphigoid 2012. Br J Dermatol 2012;167:1200-14.

16 Williams HC, Wojnarowska F, Kirtschig G et al. Doxycycline versus prednisolone as an initial treatment strategy for bullous pemphigoid: a pragmatic, non-inferiority, randomised controlled trial. Lancet 2017:389:1630-8.

17 Collin P, Salmi TT, Hervonen K, Kaukinen K, Reunala T. Dermatitis herpetiformis: a cutaneous manifestation of coeliac disease. Ann Med 2017:49:23-31.

18 Salmi TT. Dermatitis herpetiformis. Clin Exp Dermatol 2019;44: 728-31.

19 Lewis HM, Renaula TL, Garioch J] et al. Protective effect of glutenfree diet against development of lymphoma in dermatitis herpetiformis. Br J Dermatol 1996;135:363-7.

20 Hervonen K, Salmi TT, Ilus T et al. Dermatitis herpetiformis refractory to gluten-free dietary treatment. Acta Derm Venereol 2016;96:826.

Address for correspondence: Dr Emma Benton, St John's Institute of Dermatology, Guy's Hospital, Great Maze Pond, London SE1 9RT, UK.

Email: emma.benton@gstt.nhs.uk 